\title{
Protocolo alternativo para Potenciales Relacionados a Eventos Cognitivos en niños con Trastorno por Déficit de Atención y adultos con Deterioro Cognitivo
}

\author{
$\triangle$ DANIELA LóPEZ LONDOÑO ${ }^{1}$ \\ Francia Restrepo de MEjÍA ${ }^{1}$ \\ LUZ DARY RODRÍGUEZ SOTELO ${ }^{1}$
}

\section{Resumen}

Objetivo: Apoyar el diagnóstico de TDAH en niños y Deterioro cognitivo en adultos mayores, utilizando un protocolo alternativo para el estudio de Potenciales Relacionados a Eventos Cognitivos. Materiales y métodos: Construcción de un protocolo de estimulación, basado en el Paradigma Oddball en el que se utilizó carros de colores como estímulos. Este fue implementado en el software Presentation®. La actividad eléctrica cerebral producida a partir de la estimulación se registró utilizando un electroencefalograma de 32 derivaciones. Finalmente se procedió a analizar las señales obtenidas mediante la visualización de los registros. Se probó el funcionamiento del protocolo en dos sujetos de investigación de 12 años que cumplían con los criterios de inclusión y exclusión. Resultados: En dos sujetos sanos (como prueba del protocolo) se logró identificar la activación de áreas cerebrales, relacionadas a atención y memoria de trabajo. Conclusiones: Se evidenció que al aplicar el protocolo alternativo se generaron ondas cerebrales características de los procesos de atención y memoria, lo cual posibilitará la evaluación de dichas funciones en niños con TDAH y adultos mayores con Deterioro Cognitivo.

Palabras claves: Sistema de extracción de Potenciales Relacionados a Eventos, Protocolo de estimulación visual, Paradigma Oddball, Atención y memoria, Trastorno por déficit de atención (TDAH), Deterioro Cognitivo (DC).

1 Universidad Autónoma de Manizales. Manizales, Colombia.

Autor de correspondencia: López Londoño, D. (Daniela): Laboratorio de Neurofisiología "Oscar Hernán Moscoso Ariza”, Universidad Autónoma de Manizales, Antigua estación del ferrocarril, Carrera 19a \# 43 a -50, Manizales, Colombia.. Teléfono: 6 8727272, ext. 134 o 175. Correo electrónico: daniela.lopezl@autonoma.edu.co
Historia del artículo:

Artículo recibido: 18-IX-2018 / Aprobado: 30-V-2019

Disponible online: 3 de julio de 2019

Discusión abierta hasta marzo de 2021 


\title{
Alternative Protocol for Cognitive Event-Related Potentials in Children with Attention Deficit Disorder and Adults with Mild Cognitive Impairment
}

\begin{abstract}
Objective: To support the diagnosis of ADHD and Mild cognitive impairment using an alternative protocol for the study of Cognitive Event Related Potentials. Materials and methods: Construction of a stimulation protocol, based on the Oddball Paradigm, in which colored cars were used as stimuli. This was implemented in the Presentation® software. Brain electrical activity produced from the stimulation was recorded using a 32-lead electroencephalogram. Finally we proceeded to analyze the signals obtained by visualizing the records. The functioning of the protocol was tested on two 12-year research subjects who met the inclusion and exclusion criteria. Results: In two healthy subjects (as a protocol test) it was possible to identify the activation of brain areas, related to attention and working memory. Conclusions: It was evidenced that, when applying the alternative protocol, brain waves characteristic of attention and memory processes were generated; which will enable the valuation of these functions in children with ADHD and older adults with Mild cognitive impairment.
\end{abstract}

Keywords: Event-Related Potentials Extraction, Visual stimulation protocol, Oddball Paradigm, Attention and memory, Attention deficit disorder (ADHD), Mild cognitive impairment.

\section{Protocolo Alternativo para Potenciais Relacionados a Eventos Cognitivos em Crianças com Transtorno por Déficit de Atenção e idosos com Deterioração Cognitiva}

\section{Resumo}

Objetivo: Apoiar o diagnóstico de TDAH e deterioração cognitiva usando um protocolo alternativo para o estudo de Potenciais Relacionados a Eventos Cognitivos. Materiais $\boldsymbol{e}$ métodos: Construção de um protocolo de estimulação, baseado no Paradigma do Oddball, no qual carros coloridos foram usados como estímulos. Isso foi implementado no software Presentation $\circledast$. A atividade elétrica cerebral produzida a partir da estimulação foi registrada usando um eletroencefalograma de 32 derivações. Finalmente, procedemos à análise dos sinais obtidos visualizando os registros. 0 funcionamento do protocolo foi testado em dois sujeitos de pesquisa de 12 anos que preencheram os critérios de inclusão e exclusão. Resultados: Em dois sujeitos saudáveis (como teste de protocolo) foi possível identificar a ativação de áreas cerebrais relacionadas à atenção e memória de trabalho. Conclusões: Evidenciou-se que, ao aplicar o protocolo alternativo, foram geradas ondas cerebrais características dos processos de atenção e memória; o que permitirá a avaliação dessas funções em crianças com TDAH e idosos com Deterioração Cognitiva.

Palavras-chave: Potenciais relacionados com eventos sistema de extracção, Protocolo de Estimulação Visual, Paradigma Oddball, Atenção e memória, transtorno de déficit de atenção (TDAH), Deterioração cognitiva (DC). 


\section{Introducción}

El TDAH es una alteración del comportamiento, secundaria a un trastorno del desarrollo, que se manifiesta predominantemente en la infancia asociado a déficit de atención, concentración, impulsividad más frecuente que otros niños de la misma edad y nivel intelectual; todo esto observado en varios ambientes como familiares, escolares, sociales. Según el Manual de diagnóstico y estadístico de los trastornos mentales (DSM-IV, 1994) de la Asociación Americana de Psiquiatría (APA) provoca la desatención de destrezas importantes para el desarrollo personal, social, emocional, académico y físico (Pichot et al., 1995).

Por su parte, el Deterior Cognitivo leve es un síndrome que presenta un declive cognitivo mayor al esperado para su grupo de edad y escolaridad y que no afecta las actividades de la vida cotidiana. (Gauthier et al. 2006) (Mendez, L.F. 2018)

Para el estudio de Procesos Cognitivos se han utilizado los Potenciales Relacionados a Eventos (Cabrera Castro, 2011), los cuales son respuestas cerebrales cuyas ondas se modifican dependiendo de las características del estímulo (Núñez-Peña, 2004). Estos potenciales se presentan como fluctuaciones en el voltaje del Electroencefalograma (Rugg, 1996) que permiten evaluar el funcionamiento de los procesos cerebrales, mediante la identificación y análisis de ondas características desencadenadas por diferentes estímulos, generando una respuesta eléctrica en áreas cerebrales específicas y en un tiempo determinado.

Cada estimulo necesita unas características específicas para lograr activar las respuestas eléctricas que son objeto de estudio. Se ha denominado Protocolo de Estimulación a las características del estímulo tales como: modalidad de la imagen, del sonido o movimiento, tiempo de estimulación, duración del estímulo y tiempo inter-estimulo, entre otras.

Para evaluar los Potenciales Relacionados a Eventos Cognitivos, se puede utilizar cualquier tipo de estímulo sensorial, ya sea auditivo, visual, táctil o de tarea específica. Sin embargo, es necesario que exista un fenómeno de aleatoriedad y de incertidumbre para que se genere la onda P300. Por lo tanto, el Paradigma Oddball (Periáñez \& Barceló, 2004) (Katayama \& Polich, 1996) es el más utilizado para la medición de dichas funciones cognitivas.

En la evaluación clínica, se usa una tarea de discriminación visual y/o auditiva (Periáñez \& Barceló, 2004). El paradigma Oddball usa una tarea de discriminación mediante un patrón reversible en damero monocromático donde el $20 \%$ de los estímulos considerados como raros se acompañan de la presentación de un rombo pequeño en el centro de la pantalla (diana) y los estímulos comunes consisten en la presentación del patrón reversible sin estímulo diana, en el $80 \%$ restante, para un total de 200 estímulos. (Sharbrough, et al., 1991).

En investigaciones realizadas en nuestro laboratorio tales como: Modulación del componente P300 de los potenciales evocados en un grupo de niños colombianos con trastorno de atenciónhiperactividad (Restrepo et al., 2011), Efecto diferencial del subtipo clínico en los potenciales evocados cognitivos de pacientes con déficit de atención e hiperactividad (Tamayo 0, et al., 2015), se han detectado dificultades con el protocolo de estimulación visual nombrado anteriormente, por su complejidad en la comprensión de la tarea, la visualización de la imagen al ser monocromática y de escasa dimensión, genera perdida de interés causando posibles respuestas erróneas, especialmente en menores con atención dispersa por ejemplo TDAH y adultos mayores con Deterioro Cognitivo. Por lo anterior se plantea que al tener un protocolo alternativo podremos resolver problemas en la atención de los niños a la hora de evaluar los procesos cognitivos.

\section{Materiales y métodos}

Para lograr una herramienta amigable y que permita la interacción de varios componentes 
tecnológicos se propone el uso de un equipo de Electroencefalografia Cadwell con software EasyIII ® (Cadwell, 2001-2016) para la adquisición de señales eléctricas con 32 derivaciones usando el sistema internacional 10-20. Dicha señal se produce mediante la observación de un estímulo especifico generado por el software especializado de estimulación denominado Presentation $®$, el cual debe ser construido basado en especificaciones del paradigma Oddball con estimulación visual. Estas dos señales, la del amplificador y el software de estimulación son sincronizadas mediante una tarjeta de adquisición de datos National Instrument NI DAQ 6009 y proyectadas en un centro de cómputo que contiene el software Easy III 3.10., en el cual se puede observar tanto la señal EEG de los 19 canales activos y los pulsos generados por los estímulos que identifica cada una de las imágenes. Finalmente las señales son extraídas del equipo de cómputo en formato "European Data Format" ${ }^{1}$ edf para ser procesadas en sistema de visualización y análisis de las señales electroencefalografías elaborada con herramientas de software matemático MatLab®. En la Figura 1 se muestran los componentes del sistema utilizado.

\section{Protocolo de estiimulación}

Para realizar un protocolo de estimulación, se debe tener en cuenta el proceso cognitivo que se desee estudiar, así como el tipo de estímulo (imágenes, sonidos, textos, videos, movimientos, entre otros) y las características temporales, espaciales y de aleatoriedad.

En este caso en particular se realizó un protocolo de estimulación específico para la evaluación de atención y memoria en niños con TDAH y adultos con DC. Para esto se modificó el protocolo estándar oddball (patrón reversible damero) utilizando imágenes de carros verdes para los estímulos frecuentes y carros de color rojo para los infrecuentes, con un total de 200 estímulos de los cuales $20 \%$ de ellos fueron raros o infrecuentes.

\section{Figura 1. Sincronización Electroencefalograma, software Presentation ${ }^{\oplus} \mathrm{y}$ análisis de PRE}

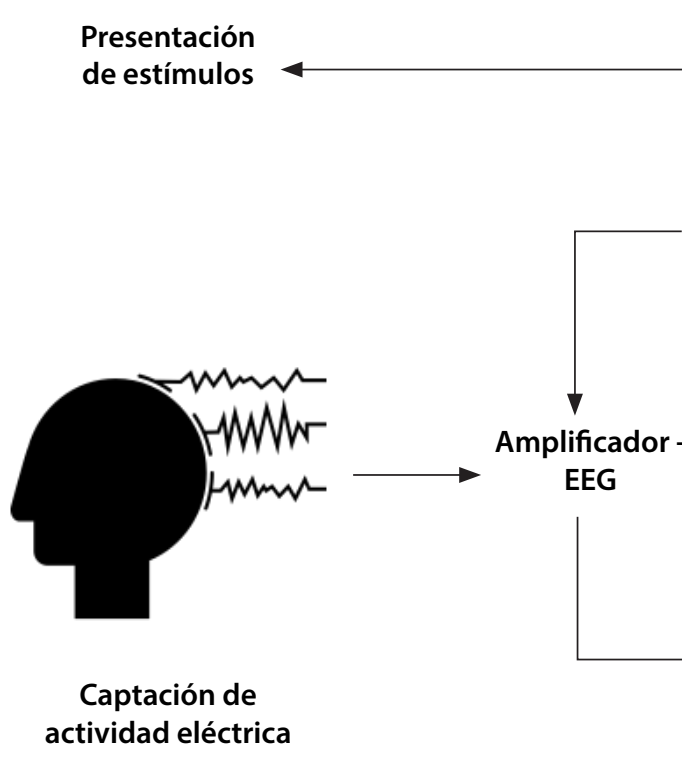

1. Laboratorio de Neurofisiología "Oscar Moscoso Ariza", Universidad Autónoma de Manizales. 
Las características temporales, espaciales y de aleatoriedad utilizadas para este protocolo fueron: $1000 \mathrm{~ms}$ para la presentación del estímulo en pantalla, sin espacio inter-estímulo entre cada una de las imágenes; además se presentó de forma aleatoria la estimulación, condicionando el programa para evitar dos estímulos raros de manera consecutiva. De acuerdo con las imágenes se escogieron dos tipos de carros con características similares en cuanto a tamaño, forma y dirección pero con diferencias discriminantes en el color como se muestra en la ilustración 1. Dichas características son utilizadas para la programación del estímulo visual, teniendo un tiempo total aproximado de tres minutos de estimulación.

\section{Potencialles a estudiar}

Los potenciales se dividen en dos: los exógenos, son respuestas que ocurren durante los primeros 200 milisegundos después de la presentación del estímulo, lo que permite evaluar el estado de la vía sensorial. Los endógenos son tardíos y se presentan después de los 200 milisegundos (NúñezPeña, 2004), además están vinculados con Procesos Cognitivos, Emocionales, de Lenguaje y Motores.

En este estudio utilizamos los potenciales exógenos enmarcados en las ondas N100, N200 y P200 y potenciales endógenos como la P300. La onda N100 indica la excitación de la vía de procesamiento de la información de la corteza primaria; está relacionada con procesos iniciales de recepción de estímulos (Tamayo-Orrego et al., 2015)a study was performed on of event-related potentials (ERPs. Así mismo la onda N200 está relacionada con el proceso cognitivo de distinción e identificación de estímulos (Castro Cabrera, 2011). La onda P200 refleja la relevancia que le da el sujeto al estímulo independientemente de la tarea encomendada.

Finalmente la onda P300 es relacionada con las tareas discriminativas con diferentes modalidades de estímulos, esta presenta una ubicación en el lóbulo parietal medio, activando áreas de asociación parietal (Martín González, 2012; Picton, 1996). Este componente se puede dividir en P3a relacionada con la atención durante el procesamiento de la tarea, y la P3b relacionada con procesamientos de la memoria (Polich, J., 2007).

\section{Toma de los Potenciales Relaciona-} dos a Eventos

- Preparación del sujeto de investigación: Previo a la toma de toma de los potenciales se realiza la ubicación de electrodos acorde con el sistema internacional 10/20 (Klem et al., 1999); el sujeto debe estar sentado en una posición cómoda, frente a una pantalla de 22 pulgadas que se encuentra a un metro de distancia, en la cual se presentan los estímulos anteriormente descritos. Debe evitarse movimiento que altere la señal.

Figura 2. Imágenes utilizadas para la estimulación

Modificada de http://pixers.es/vinilos/vector-conjunto-de-coches-de-dibujos-animados-retro-29231633

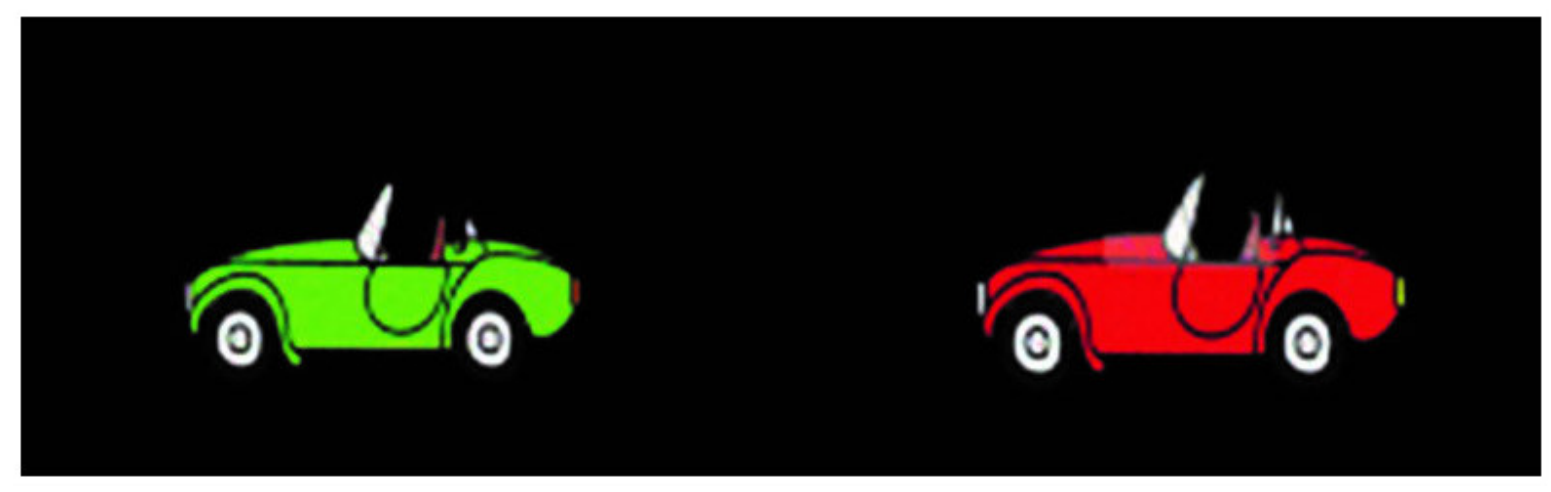




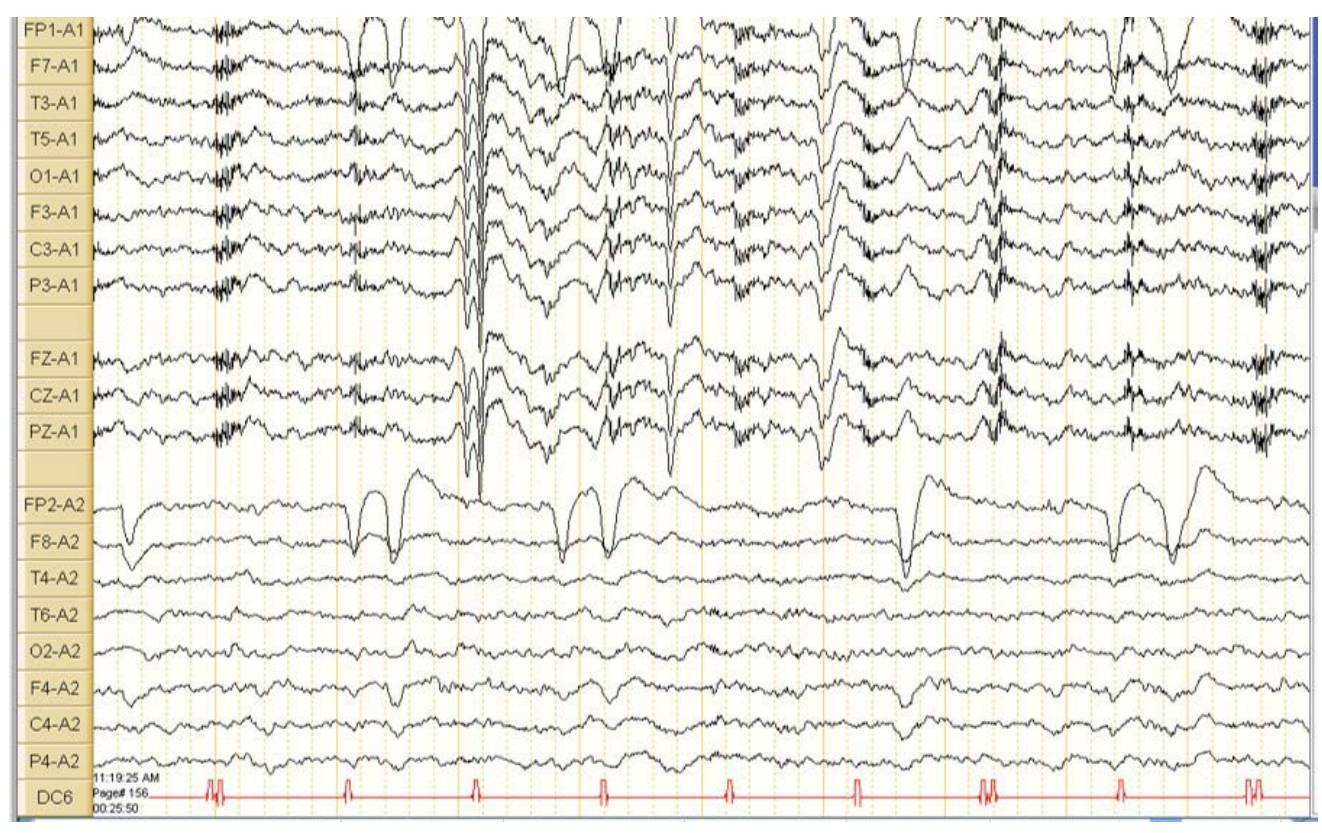

- $\quad$ Registro electroencefalográfico (EEG): La señal obtenida por los electrodos es visualizada en un equipo de recepción de datos (equipo de cómputo con el software EasyIII). Por otra parte el sistema de interfaz entre dicho software y el software de estimulación Presentation ${ }^{\circledR}$ capta la señal de los estímulos generados y los traduce en una representación gráfica en forma de pulsos cuadrados, que quedan registrados en el equipo de recolección de datos, de allí se extrae la sincronización de los pulsos junto con los registros de electroencefalografía, que se presentan durante la estimulación como se muestra en la ilustración 2.

Para un adecuado experimento es necesario que éstos tengan sincronización precisa y rendimiento.

Una vez adquiridas las señales electroencefalográficas son extraídas y promediadas para obtener los Potenciales Relacionados a Eventos Cognitivos. Se realiza la caracterización de señales que permite visualizar información relevante de las ondas, apo- yando el estudio de los procesos cognitivos, normales o patológicos (atención / memoria).

\section{Pilotaje para la prueba del protocolo} alternativo para Potenciales Relacionados a Eventos Cognitivos

Previo a la toma de los potenciales: se diligenció el consentimiento a los acudientes y asentimiento a los menores ${ }^{2}$ y se realizó un entrenamiento a la actividad a realizar.

Los sujetos fueron un hombre y una mujer, con las siguientes características: edad 12 años, con similar nivel socio económico, educación, prevalencia y preferencia diestra y sin patologías, puesto que las pruebas se deben realizar en sujetos sanos para determinar el buen funcionamiento del protocolo puesto que para ellos existen rangos de normalidad de los valores de las ondas ya establecidos en la literatura.

2. En concordancia con el capítulo II artículo 17 de la resolución 8430 de 1993, se consideró de riesgo mínimo. Proyecto aprobado por el comité de Bioética de la Universidad Autónoma de Manizales (UAM), según consta en el acta No 071-sep 13-2017. 


\section{Resultados y discusión}

Los investigadores (Tamayo O. et al., 2015) (Restrepo et al., 2011) realizaron un estudio de Potenciales Relacionados a Eventos, donde evidenciaron que una amplitud menor de $2 \mu \mathrm{V}$ en niños normales que realizan una actividad cognitiva, puede deberse a ruido y generar confusiones.
Por dicha razón para el análisis de la actividad eléctrica en el presente artículo se trabajó con amplitudes de $2 \mu \mathrm{V}$ mínimo.

En la Tabla 1, en la primera columna se observan los electrodos utilizados; en la segunda columna amplitud y latencia de la N100 y así sucesivamente con las ondas P100, N200, P200 y P300. La NA indica que no se encontró actividad menor de $2 \mu \mathrm{V}$.

\begin{tabular}{|c|c|c|c|c|c|c|c|c|c|c|}
\hline Género & \multicolumn{10}{|c|}{ Femenino } \\
\hline Edad & \multicolumn{10}{|c|}{12 años } \\
\hline Estrato económico & \multicolumn{10}{|c|}{ Medio medio } \\
\hline Nivel educativo & \multicolumn{10}{|c|}{ Octavo } \\
\hline Lateralidad & \multicolumn{10}{|c|}{ Diestra en prevalencia y preferencia } \\
\hline \multirow[t]{2}{*}{ Características } & \multicolumn{2}{|c|}{ N100 } & \multicolumn{2}{|c|}{ P100 } & \multicolumn{2}{|c|}{ N200 } & \multicolumn{2}{|c|}{ P200 } & \multicolumn{2}{|c|}{ P300 } \\
\hline & $\mathbf{L}$ & A & $\mathbf{L}$ & A & $\mathbf{L}$ & A & $\mathbf{L}$ & A & $\mathbf{L}$ & A \\
\hline Fz & 156 & 4 & 96 & 3 & 264 & 3 & NA & NA & 356 & 3 \\
\hline $\mathbf{C z}$ & 136 & 3 & NA & NA & NA & NA & NA & NA & 345 & 4 \\
\hline $\mathbf{P z}$ & 156 & 4 & NA & NA & NA & NA & NA & NA & 348 & 4 \\
\hline Fp1 & 160 & 4 & NA & NA & 228 & 7 & NA & NA & 348 & 3 \\
\hline Fp2 & 164 & 4 & NA & NA & 245 & 6 & NA & NA & 337 & 2 \\
\hline F7 & NA & NA & NA & NA & 237 & 3 & NA & NA & 357 & 2 \\
\hline F3 & 156 & 3 & 96 & 3 & 240 & 3 & NA & NA & 344 & 3 \\
\hline F8 & NA & NA & NA & NA & 236 & 3 & NA & NA & NA & NA \\
\hline F4 & 164 & 4 & NA & NA & NA & NA & NA & NA & 332 & 2 \\
\hline T3 & NA & NA & NA & NA & 192 & 3 & NA & NA & 372 & 2 \\
\hline T4 & NA & NA & NA & NA & 204 & 4 & NA & NA & 360 & 4 \\
\hline T5 & NA & NA & 149 & 2 & NA & NA & 225 & 2 & NA & NA \\
\hline T6 & NA & NA & 144 & 2 & 204 & 3 & 240 & 5 & 352 & 3 \\
\hline C3 & 156 & 4 & NA & NA & NA & NA & NA & NA & NA & NA \\
\hline C4 & 153 & 3 & 100 & 5 & 232 & 2 & NA & NA & 348 & 4 \\
\hline P3 & 129 & 3 & NA & NA & NA & NA & 257 & 2 & 337 & 3 \\
\hline P4 & 133 & 2 & NA & NA & NA & NA & 257 & 2 & 373 & 3 \\
\hline 01 & 132 & 2 & 148 & 7 & 204 & 4 & NA & NA & 329 & 4 \\
\hline 02 & NA & NA & 144 & 5 & 200 & 3 & NA & NA & 333 & 3 \\
\hline
\end{tabular}




\begin{tabular}{|c|c|c|c|c|c|c|c|c|c|c|}
\hline Género & \multicolumn{10}{|c|}{ Masculino } \\
\hline Edad & \multicolumn{10}{|l|}{12} \\
\hline Estrato económico & \multicolumn{10}{|c|}{ Medio medio } \\
\hline Nivel educativo & \multicolumn{10}{|c|}{ Octavo } \\
\hline Lateralidad & \multicolumn{10}{|c|}{ Diestra en prevalencia y preferencia } \\
\hline Características & \multicolumn{2}{|c|}{ N100 } & \multicolumn{2}{|c|}{ P100 } & \multicolumn{2}{|c|}{ N200 } & \multicolumn{2}{|c|}{ P200 } & \multicolumn{2}{|c|}{ P300 } \\
\hline & $\mathbf{L}$ & A & $\mathbf{L}$ & A & $\mathbf{L}$ & A & $\mathbf{L}$ & A & $\mathbf{L}$ & A \\
\hline $\mathbf{F z}$ & 96 & 2 & 88 & 2 & NA & NA & NA & NA & 269 & 1 \\
\hline $\mathbf{C z}$ & 88 & 1 & 96 & 1 & 180 & 2 & 229 & 1 & 269 & 1 \\
\hline $\mathbf{P z}$ & 92 & 2 & 96 & 0,7 & 184 & 4 & 245 & 2 & 270 & 2 \\
\hline Fp1 & NA & NA & NA & NA & 240 & 2 & NA & NA & 268 & 2 \\
\hline Fp2 & NA & NA & NA & NA & 244 & 4 & NA & NA & NA & NA \\
\hline F7 & 88 & 1 & 96 & 1 & 220 & 2 & NA & NA & 273 & 1 \\
\hline F3 & 88 & 1 & 96 & 1 & 180 & 2 & NA & NA & 269 & 1 \\
\hline F8 & 96 & 2 & NA & NA & 185 & 4 & NA & NA & 325 & 5 \\
\hline F4 & 96 & 2 & NA & NA & 184 & 4 & NA & NA & 376 & 10 \\
\hline T3 & NA & NA & 96 & 2 & 200 & 2 & NA & NA & NA & NA \\
\hline T4 & NA & NA & NA & NA & 204 & 5 & NA & NA & 376 & 7 \\
\hline T5 & NA & NA & 96 & 2 & NA & NA & 245 & 6 & NA & NA \\
\hline T6 & NA & NA & 124 & 3 & 197 & 1 & 244 & 6 & 376 & 5 \\
\hline C3 & 88 & 2 & NA & NA & 180 & 2 & 244 & 2 & NA & NA \\
\hline C4 & NA & NA & NA & NA & 204 & 5 & NA & NA & 376 & 10 \\
\hline P3 & 88 & 2 & NA & NA & 200 & 2 & 244 & 4 & NA & NA \\
\hline P4 & 92 & 2 & NA & NA & 200 & 6 & NA & NA & 376 & 10 \\
\hline 01 & 124 & 1 & 152 & 8 & 221 & 2 & 244 & 2 & 268 & 3 \\
\hline 02 & 92 & 1 & 124 & 2 & NA & NA & NA & NA & 364 & 6 \\
\hline
\end{tabular}

En la Tabla 2, en la primera columna se observan los electrodos utilizados; en la segunda columna amplitud y latencia de la N100 y así sucesivamente con las ondas P100, N200, P200 y P300. La NA indica que no se encontró actividad menor de $2 \mu \mathrm{V}$.

N100: Aparece entre los 70-200ms con una amplitud de 2-4 $\mu \mathrm{V}$ después de presentado el es- tímulo y evidencia la excitación de la vía nerviosa utilizada.

Se observó en áreas primarias somato sensoriales, áreas de asociación y en la área multimodal parieto- temporo-occipital en ambos hemisferios. De igual manera se activaron las áreas encargadas de la ejecución y memoria espacial extra-corporal. 
A pesar de que la onda N100 se considera como recepción del estímulo la conexión con áreas de asociación temprana, puede deberse a la permanencia del estímulo. (Redolar Ripoll, 2014).

P100: Su aparición se da alrededor de los $100 \mathrm{~ms}$, la amplitud de la P100 se disminuye a medida que aumentan los estímulos; es generada por la diferenciación del mismo; esta corresponde a una respuesta sensorial visual y se encuentra en la región occipital (Chiappa, 1997). En nuestro estudio se presenta en áreas de asociación somato sensorial derecha, áreas primarias visuales y áreas de asociación visuales temporo-occipitales.

N200: Se observa entre 180 y 325 ms, corresponde a la identificación y evaluación del estímulo en áreas primarias somato sensoriales y visuales; áreas de asociación temporales y frontales bilateralmente, relacionadas con asociación visual, atención y memoria de trabajo.

P200: Esta onda se observa después de la aparición de la N100 alrededor de los 100 y 200ms, muestra la relevancia del estímulo infrecuente o raro. Refleja la relevancia que le da el sujeto al estímulo, no se evidencio en ninguna área primaria; si hay presencia de la onda en áreas de asociación temporales y parietales multimodales.

P300: Se presenta con una amplitud de 4-6 $\mu \mathrm{V}$ en electrodos centrales, esta onda permite la discriminación de la tarea, la cual puede variar por la dificultad de la misma. La P300 en muchas ocasiones puede evidenciarse como P3a que refleja la atención durante el proceso de la tarea y la P3b que manifiesta la memoria. Esta onda se encuentra con picos de latencia máxima alrededor de los 300 a 350ms en adultos jóvenes normales (Picton, 1992) (Polo, et al., 1999); la latencia de la P300 aumenta entre 1 y 1.8 ms después de los 20 años (Neshige \& Lüders, 1988). Se encontró en áreas primarias somato-sensoriales y áreas visuales, además de áreas de asociación frontales dorso lateral encargadas de la atención y memoria de trabajo; en el lóbulo temporal con áreas de asociación visual y emociones visuales.

\section{Conclusiones}

Fue posible construir y poner en ejecución un protocolo para la evaluación de Potenciales Relacionados a Eventos Cognitivos modalidad visual, utilizando tecnología de bajo costo y fácil realización. En este, se evidenció y detectó la activación de áreas cerebrales y su relación con la función generada por los estímulos visuales. Al poder utilizar más de tres electrodos como tradicionalmente se había hecho, se tiene la posibilidad de realizar estudios funcionales del cerebro más amplios, que permiten profundizar en el conocimiento de procesos cognitivos.

El uso de imágenes (en este caso carritos de colores) facilita a los niños con TDAH y adultos mayores con Deterioro Cognitivo la aceptación de la prueba, pues se asemeja más a una actividad lúdica, a diferencia del uso de imágenes abstractas como el damero monocromático como se realiza tradicionalmente. En los resultados de este pilotaje, el protocolo alternativo permitió observar consistencia en la generación de las ondas y activación de las áreas cerebrales. Sin embargo, aun esta muestra es muy pequeña (dos sujetos de investigación), por lo cual como trabajo futuro se requiere una población mayor.

Finalmente, observamos que protocolos de este tipo pueden ser utilizados en otras investigaciones con variaciones en la modalidad de estímulo como el auditivo o procesos cognitivos diferentes, entre los que se encuentra el lenguaje y las emociones.

\section{Agradecimientos}

Agradecemos a la Universidad Autónoma de Manizales por financiar el recurso humano y los insumos; facilitar el espacio y las herramientas tecnológicas para la construcción y evaluación de protocolos de estimulación mediante el sistema SIPRES.

\section{Conflicto de intereses y financiación}

Los autores manifiestan que no presentan ningún conflicto de intereses. 


\section{Financiación}

Universidad Autónoma de Manizales.

\section{Referencias}

Cabrera Castro , P. A., 2011. Extracción y selección de caracteristicas discriminantes para la detección de TDAH en registros de potenciales evocados cognitivos = Extraction and selection of discriminative features for ADHD detection on cognitive evoked potentials recording. Universidad Nacional de Colombia-Sede Manizales.

Cadwell, 2001-2016. cadwell (R). [En línea] Available at: https://www.cadwell.com/products/

Carretié, L., Iglesias, J., Garcia, T. \& Ballesteros, M., 1997. N300, P300 and the emotional processing of visual stimuli. Electroencephalography and clinical Neurophysiology 103(2), pp. 298-303.

Chiappa, k., 1997. Evoked Potentials in Clinical. Philadelphia New York: Liooincott-Raven.

Gauthier, S., MD, Reisberg, B., MD, Zaudig, M., MD, Petersen, R. C., MD, Ritchie, K., PhD, Broich, K., PhD, ... Hampel, H., PhD. (2006). Mild cognitive impairment. The Lancet, 367(9527), 1979. doi:10.1016/ s0140-6736(06)68881-8

Katayama, J. \& Polich , J., 1996. P300 from one-, two-, and three-stimulus auditory paradigms. International Journal of Psychophysiology, pp. 23(1) 33-40.

Klem , G. H., Luders, H. O., Jasper, H. H. \& Elger , C., 1999. The ten-twenty electrode system of the international The International Federation of Clinical Neurophysiology. Electroencephalography and Clinical Neurophysiology, pp. Supplement 52, 3-6.

Martín González, L. (2012). Desarrollo de un sistema Brain Computer Interface basado en potenciales evocados P300 para el control de dispositivos domóticos. Retrieved from http://uvadoc.uva.es/ handle/10324/2655

Mendez, L.F. (2018). Influencia de los factores sociodemográficos y el desempeño de las actividades instrumentales de la vida cotidiana en en el envejecimiento normal y el deterioro cognitivo.

Neshige, R. \& Lüders, H., 1988. Identification of a negative bitemporal component (N300) of the event-related potentials demonstrated by noncephalic recordings. Neurology, 38(11), pp. 1803-1803.
Núñez-Peña, M. I. C. M. J. \&. E. C., 2004. Potenciales evocados cerebrales en el contexto de la investigación psicológica: una actualización. Anuario de psicología, 35(1), pp. 3-22.

Periáñez, J. \& Barceló, F., 2004. Electrofisiología de las funciones ejecutiva. Revista de neurología, p. Vol. 38 no 2009.

Pichot, P., López-Ibor Aliño, J. \& Valdés Miyar, M., 1995. DSM-IV Manual diagnóstico y estadístico de los trastornos mentales. En: s.l.:MASSON.

Picton, T. W., 1992. The P300 wave of the human eventrelated potential. Journal of clinical neurophysiology 9, pp. 456-479.

Policg,J., 200 . P300 as a clinical assay: rationale,evaluation and findings. International Journal of Psychophysiology , pp. 38(1), 3-22.

Polich, J., 2007. Updating P300: an integrative theory of P3a and P3b. Clinical Neurophysiology, Volumen 118, p. 2128-2148.

Redolar Ripoll, D., 2014. Neurociencia Cognitiva. Catalunya Barcelona: Panamericana.

Restrepo, F. y otros, 2011. Modulación del componente P300 de los potenciales evocados en un grupo de niños colombianos con trastorno de atención-hiperactividad. Acta Neurológica Colombiana, pp. 146153.

Rugg, M. \&. C. M., 1996. Electrophysiology of Mind: Eventrelated Brain Potentials and Cognition.. Oxford University Press..

Sharbrough, F. y otros, 1991. American Electroencephalographic society guidelines for standard electrode position nomenclature. Journal Clinical Neurophysiology, pp. 8(2) 200-2.

Tamayo O, L. y otros, 2015. Efecto diferencial del subtipo clínico en los potenciales evocados cognitivos de pacientes con déficit de atención e hiperactividad. Revista Colombiana de Psiquiatría, pp. 77-86.

\section{PARA CITAR ESTE ARTÍCULO / TO REFERENCE THIS ARTICLE / PARA CITAR ESTE ARTIGO /}

López Londoño, D.; Restrepo de Mejía, F.; Rodríguez Sotelo, L.D.(2019). Protocolo alternativo para Potenciales Relacionados a Eventos Cognitivos en niños con Trastorno por Déficit de Atención y adultos con Deterioro Cognitivo. Revista EIA, 16(32), Julio-Diciembre, pp. 243-252. [Online]. Disponible en: https://doi.org/10.24050/reia.v16i32.1264 